\title{
Dynamic regimes of buoyancy-affected two-phase flow in unconsolidated porous media
}

\author{
M. Stöhr ${ }^{1, *}$ and A. Khalili ${ }^{1,2, \dagger}$ \\ ${ }^{1}$ Max Planck Institute for Marine Microbiology, Celsiusstrasse 1, D-28359 Bremen, Germany \\ ${ }^{2}$ International University Bremen, Campus Ring 1, D-28759 Bremen, Germany
}

(Received 15 June 2005; published 1 March 2006)

\begin{abstract}
The invasion and subsequent flow of a nonwetting fluid (NWF) in a three-dimensional, unconsolidated porous medium saturated with a wetting fluid of higher density and viscosity have been studied experimentally using a light-transmission technique. Distinct dynamic regimes have been found for different relative magnitudes of viscous, capillary, and gravity forces. It is shown that the ratio of viscous and hydrostatic pressure gradients can be used as a relevant dimensionless number $K$ for the characterization of the different flow regimes. For low values of $K$, the invasion is characterized by the migration and fragmentation of isolated clusters of the NWF resulting from the prevalence of gravity and capillary forces. At high values of $K$, the dominance of viscous and gravity forces leads to an anisotropic fingerlike invasion. When the invasion stops after the breakthrough of the NWF at the open upper boundary, the invasion structure retracts under the influence of gravity and transforms into stable vertical channels. It is shown that the stability of these channels is the result of a balance between hydrostatic and viscous pressure gradients.
\end{abstract}

DOI: 10.1103/PhysRevE.73.036301

PACS number(s): 47.56.+r, 47.20.Bp, 47.55.Kf, 47.80.-v

\section{INTRODUCTION}

Immiscible two-phase flow in porous media is an active field of research due to its large number of applications, like, e.g., in the areas of petroleum engineering (enhanced oil recovery), chemical engineering (gas-liquid flow in packed columns), hydrology (spread and fate of contaminants), or marine science (migration and escape of gas in permeable sediments). The field further received considerable attention from theoretical physicists since the observed phenomena were found to be manifestations of various theoretical concepts such as fractals [1], percolation [2], kinetic roughening [3], self-organized criticality [4], and quenched disorder [5]. In the following we summarize the main concepts relevant to our work. For a more comprehensive review of the numerous experimental, numerical, and theoretical studies see $[6,7]$.

The dynamics of the interface between two immiscible fluids in a porous medium is governed by the interplay between capillary, viscous, and gravity forces. Consequently, the phenomena are aptly described by a set of dimensionless numbers representing the relative magnitude of these forces at the pore scale. A common choice is the combination of the bond number Bo and the capillary number $\mathrm{Ca}$. The former is defined as the ratio of gravity and capillary forces

$$
\text { Bo }=\frac{\Delta \rho g d^{2}}{\sigma},
$$

where $\Delta \rho=\rho_{1}-\rho_{2}$ is the difference of fluid densities, $g$ is the acceleration of gravity, $d$ is the pore diameter, and $\sigma$ is the interfacial tension. The indices 1 and 2 denote the invading and defending fluid, respectively. The capillary number

\footnotetext{
*Present address: German Aerospace Center, Institute of Combustion Technology, Pfaffenwaldring 38-40, D-70569 Stuttgart, Germany. Electronic address: michael.stoehr@dlr.de

†Electronic address: akhalili@mpi-bremen.de
}

$$
\mathrm{Ca}=\frac{v \mu}{\sigma}
$$

represents the relation of viscous to capillary forces, where $v$ is a characteristic velocity and $\mu$ is the dynamic viscosity of the more viscous fluid. Consideration of the viscosity ratio $M=\mu_{1} / \mu_{2}$ is necessary when viscous forces play a role in both fluids. Viscous forces can either stabilize $(M>1)$ or destabilize $(M<1)$ the interface. Similarly, the relative arrangement of two fluids with different densities can lead to stabilization $(\mathrm{Bo}>0)$ or destabilization $(\mathrm{Bo}<0)$.

The present study investigates the combination of viscous $(M<1)$ and density $(\mathrm{Bo}<0)$ instabilities in a threedimensional unconsolidated porous medium. A nonwetting fluid (NWF) invading at the bottom of the medium is less dense and less viscous than the displaced wetting fluid (WF).

In the absence of gravity $(\mathrm{Bo}=0)$, the prevalence of capillary forces at small $\mathrm{Ca}$ leads to a process known as capillary fingering $(\mathrm{CF})$. When viscous forces dominate at high $\mathrm{Ca}$, the displacement pattern is characterized by an instability referred to as viscous fingering (VF). The patterns of both $\mathrm{CF}$ and VF have been reproduced numerically with the algorithms of invasion percolation (IP) and diffusion-limited aggregation (DLA), respectively. The characterization of CF-IP and VF-DLA in terms of their fractal dimension has been accomplished numerically and experimentally and through theoretical scaling arguments [6].

Most practical issues involve three-dimensional systems with fluids of different density and therefore require the effect of gravity to be taken into account. In the stabilizing configuration $(\mathrm{Bo}>0)$, where the less dense fluid invades from the top, gravity reduces the vertical extension of the interface. It can thereby counteract or prevent the VF instability $[8,9]$. Experimental and numerical studies of the destabilizing configuration $(\mathrm{Bo}<0)$ have shown a vertical growth of the interface in the form of single branches or fingers 


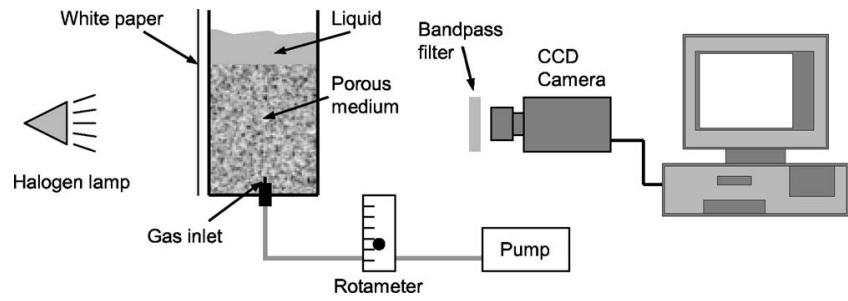

FIG. 1. Setup of the light-transmission technique used for the visualization of gas inside a porous medium.

similar to the VF instability $[10,11]$. The analysis of these branches has confirmed the scaling of a characteristic length scale $\xi$, as a measure of their transverse width, according to the power law $\xi \propto|\mathrm{Bo}|^{-0.47}$ predicted from percolation theory [12]. A second characteristic length is the vertical distance $h$ where the hydrostatic pressure difference equals the capillary pressure, $h=|\mathrm{Bo}|^{-1} d$. If the vertical extension of a NWF cluster exceeds $h$, it can independently migrate upwards. Such migration processes, which often coincide with fragmentation (snapoff) of the NWF, have been studied by $[10,13,14]$.

While these processes of slow drainage, governed by the combination of capillary and destabilizing gravity forces, have been rather well investigated, hardly any information is available for the case when the additional viscous instability becomes important for higher $\mathrm{Ca}$. Furthermore, most of the previous studies merely aim at the invasion process of the NWF-i.e., its dynamics until breakthrough at the boundary of the porous medium. The subsequent flow through the porous medium, which is important for practical issues such as the flow of hydrocarbons through oil reservoirs or the seepage of gas through marine sediments, has received little attention.

In the present work the combination of viscous and density instabilities is studied experimentally. Distinct dynamic regimes are observed over a range of values for $\mathrm{Ca}$. A fundamental transition in the behavior after breakthrough at the boundary has been found. It is shown that the ratio of viscous and hydrostatic pressure gradients is a relevant dimensionless number for the characterization of the observed phenomena.

After the description of the experimental setup in Sec. II, the presentation and discussion of the results are divided into two parts. Section III deals with the invasion of the NWF until breakthrough at the upper boundary. The fundamental changes in the dynamics of the NWF at breakthrough and the phenomenology of the subsequent flow through the porous medium are the subject of Sec. IV. A summary and conclusions are given in Sec. V.

\section{EXPERIMENTAL METHOD}

A light-transmission technique as sketched in Fig. 1 was employed to visualize the distribution of a NWF inside a three-dimensional porous medium. The porous medium is contained in a transparent box with a square base of 96 $\times 96 \mathrm{~mm}^{2}$ inner dimensions. The height of the porous medium is $H=13 \mathrm{~cm}$. Air as a NWF is injected at different flow rates $Q$ through a nozzle ( $2 \mathrm{~mm}$ inner diameter) at the center
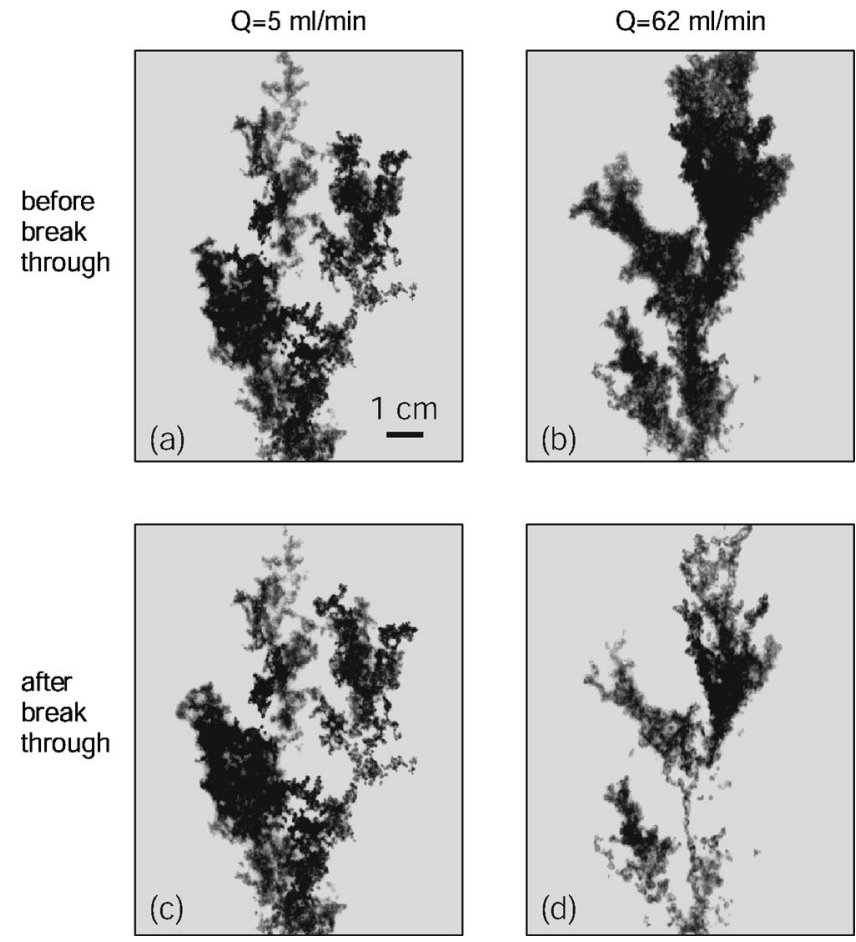

FIG. 2. Distribution of gas in the porous medium directly before (a),(b) and $\Delta t=1.5 \mathrm{~s}$ after (c),(d) breakthrough at the upper boundary. For the flow rate $Q=5 \mathrm{ml} / \mathrm{min}$ (a),(c), the dynamics is characterized by a continual migration of isolated gas clusters, eventually leading to the formation of a cone-shaped area of trapped bubbles as shown in Fig. 6(a). For $Q=62 \mathrm{ml} / \mathrm{min}$, the invading gas forms an entirely connected, fingerlike structure until breakthrough (b). Then it retracts (d) and transforms into a single stable channel [Fig. 6(b)].

of the base. This geometry was chosen in order to prevent the NWF from reaching the side walls. The injection was driven by a pump which delivered a constant pressure of $p$ $=140$ mbar. The flow rate was measured and controlled by a rotameter with an integrated valve. Due to changes in permeability of the porous medium during invasion, the pressure at the nozzle and accordingly the air injection rate fluctuate during an experiment. From measurements of the pressure fluctuations at the nozzle ( $\Delta p<10 \mathrm{mbar})$, the fluctuations of air injection were estimated to be below $10 \%$.

A sheet of white paper, attached on one side of the box, is illuminated by a $20-\mathrm{W}$ halogen lamp at a distance of $50 \mathrm{~cm}$. From the opposite side, a charge-coupled-device (CCD) camera records the two-dimensional (2D) projection of the 3D air distribution, which reveals itself by its shadow as shown, e.g., in Fig. 2. An image sequence with a framerate of $10 \mathrm{fps}$ and a resolution of $640 \times 384$ pixels is continuously transferred to a PC and stored on its harddisc.

The preparation of a both saturated and rigid 3D porous medium is an experimental challenge. While the rigidity can be attained, e.g., by sintering of packed glass beads, complete saturation of the medium is then hard to realize. In the present work, an unconsolidated packing of glass beads with sizes in the range of $0.5-2 \mathrm{~mm}$ has been used. Here complete saturation can be easily attained by pouring the loose packing into the box filled with liquid and stirring the mixture so that all trapped gas can escape. During the experi- 
ments, the open top of the medium is overlaid by a $3-\mathrm{cm}$ layer of the WF. Although the packing has been thoroughly compressed before each experiment using a perforated plate, the displacement of individual grains in consequence of forces exerted by the fluids cannot be totally precluded.

A prerequisite for this method to work is that the porous medium, when it is saturated with the WF, behave optically homogeneous. As in previous studies like, e.g., [10,15], this was achieved by the use of transparent solids and liquids with the same index of refraction, $n$. The porous matrix is made of randomly packed grains of broken glass (Boroclear, $n=1.472$ ). The WF is a mixture of two Dow Corning silicone oils: namely, DC $550(n=1.49)$ and DC $556(n=1.46)$. The mixing ratio is adjusted so that the mixture matches the refractive index of Boroclear. It has then a density of $\rho$ $=1010 \mathrm{~kg} / \mathrm{m}^{3}$, a viscosity of $\mu=52 \times 10^{-3} \mathrm{~Pa} \mathrm{~s}$, and a surface tension of $\sigma=20 \times 10^{-3} \mathrm{~N} / \mathrm{m}$.

Generally, the refractive indices of two materials can be matched at only one wavelength. In a system composed of a granular material and a liquid only the light with this wavelength propagates straightly, while for other parts of the spectrum the paths become more and more distorted with growing distance from the matched wavelength $\lambda$. Here the refractive indices were matched at $\lambda=650 \mathrm{~nm}$ using a spectrophotometer as described by [15] and an interference bandpass filter with a transmission range of 610 $\mathrm{nm}<\lambda<690 \mathrm{~nm}$ is used to restrict the imaging to the spectral range with minimal distortion.

With an estimated pore diameter of $d=0.5 \mathrm{~mm}$ and $\rho_{\text {air }}$ $=1.225 \mathrm{~kg} / \mathrm{m}^{3}$, the value of the bond number (1) is $\mathrm{Bo}=$ -0.12 for all experiments. Using $\mu_{\text {air }}=1.8 \times 10^{-5} \mathrm{~Pa} \mathrm{~s}$, the viscosity ratio is $M=3.5 \times 10^{-4}$. The definition of the capillary number $\mathrm{Ca}$ according to (2) is ambiguous because the velocity $v$ varies in space and time. For the actual calculation of $\mathrm{Ca}$ from experiments several specific definitions have been used in the literature (see, e.g., $[16,17,10])$. For the discussion of the invasion process in Sec. III, we provide the ranges of Ca based on the mean upward velocity of the upper tip of the invading gas. The discussion of the subsequent flow of gas through the porous medium will make clear that the value of $\mathrm{Ca}$ is then no longer significant, and therefore a new appropriate dimensionless number $K$ will be defined in Sec. IV.

The obtained images are first subject to a number of preprocessing transformations in order to separate the structure of the distributed gas from the background. The image data further allow for a rough estimation of the total content of gas in the flow cell. Since the gas reduces the transmissivity of the medium and appears in the images $g(x, y, t)$ as its (darker) shadow, the difference of the sums of gray values,

$$
G(t)=\sum_{x=1}^{384} \sum_{y=1}^{640} g(x, y, t=0)-\sum_{x=1}^{384} \sum_{y=1}^{640} g(x, y, t),
$$

is a proxy for the total amount of gas. However, due to the overlapping in the $2 \mathrm{D}$ projection, an exact quantitative relation cannot be made. (a)
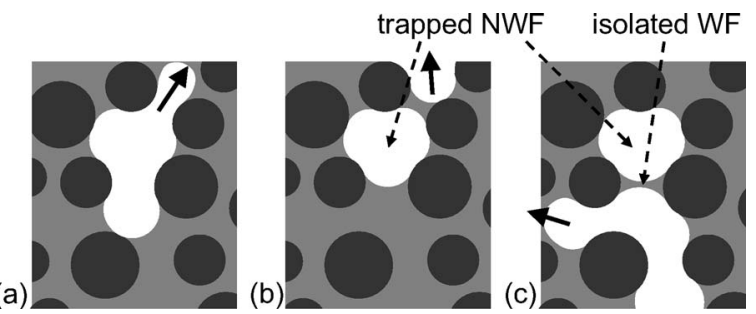

FIG. 3. Mechanisms of buoyancy-driven migration of isolated gas clusters. During upward migration (a), the cluster can break up into fragments, which partly become trapped (b). The trapped bubbles can block the respective pore and thereby force following clusters coming from below to circumvent this pore (c). The repeated occurrence of these processes leads to the cone-shaped area of trapped bubbles shown in Fig. 6(a).

\section{INVASION}

A series of 31 experiments has been performed where air as a NWF was injected with different flow rates at the bottom of the saturated porous medium and the dynamics of the NWF has been visualized as described in Sec. II. The flow rates covered a range of $3<Q<186 \mathrm{ml} / \mathrm{min}$ corresponding to $0.01<\mathrm{Ca}<0.11$. This section discusses the invasion of the gas-i.e., its dynamics before breakthrough at the upper boundary. Depending on the flow rate, the observed phenomenologies can be divided into two distinct regimes, as described in the following.

\section{A. Isolated clusters}

For flow rates lower than $Q=30 \mathrm{ml} / \mathrm{min}(\mathrm{Ca}<0.06)$, the spatial structure of gas before breakthrough is characterized by a distributed set of isolated clusters. A representative example is shown in Fig. 2(a). The structures evolved from repeated fragmentation and migration processes. When the injected cluster at the inlet has reached a certain vertical extension $h$, the effect of buoyancy exceeds the pinning capillary forces and the cluster begins to move upward independently. The comparison of the hydrostatic pressure drop $\Delta \rho g h$ with the capillary pressure $2 \sigma / d$ leads to an estimate for $h$ which is inversely proportional to the bond number, $h \approx|\mathrm{Bo}|^{-1} d$. For the present experiments this is equivalent to a height of a few millimeters. The migrating cluster may further break up into fragments until it eventually stops or escapes at the upper boundary.

Fragmentation, migration, and coalescence of NWF clusters have been studied extensively for 2D systems both experimentally and numerically $[13,14,17-19]$. In the present 3D experiments, additional buoyancy-affected phenomena have been observed which we term "blocking" and "circumvention": As is outlined in Fig. 3, a cluster may fragment during migration, and the remaining lower part will then get trapped preferably in a larger pore. If then another cluster arrives from below, it may be more favorable that it circumvents the trapped cluster rather than remobilizing it. A coalescence of the two clusters can be inhibited if the WF between the NWF clusters is isolated from the bulk of the WF as sketched in Fig. 3(d). Through these mechanisms the 
trapped NWF cluster thus blocks the respective pore and prevents clusters coming from below from following the same path upward. Consequently the upward migrating clusters follow different individual paths, and the repeated processes of blocking and circumvention lead to the coneshaped distribution of trapped bubbles observed here and in Sec. IV B.

The above findings are in some way contradictory to the experimental results from 3D systems of [10], who report on coalescence of migrating clusters with trapped gas rather than circumvention. Correspondingly they found straight vertical configurations instead of the cone-shaped structures described here. A potential explanation is the considerably lower flow rate used in their experiments. If the isolated WF has enough time to escape through film flow [14], a coalescence and subsequent remobilization may be favored compared to circumvention.

\section{B. Connected fingerlike structures}

For flow rates higher than $Q=30 \mathrm{ml} / \mathrm{min}(\mathrm{Ca}>0.06)$, the injected gas remains entirely connected until it escapes at the upper boundary. An example of such a structure is shown in Fig. 2(b). The evolving structures are characterized by a hierarchical arrangement of growing fingers. The initially linear increase in Fig. 4(b) indicates that the gas enters the medium with a constant rate until breakthrough. The main body evolves vertically from the inlet towards the upper boundary. On all scales smaller fingers start growing laterally with a preferably upward inclination.

The observed structures resemble in some way those of VF processes. Experimental investigations of VF have to preclude the influence of gravity and are therefore typically limited to horizontal 2D systems. Few experiments and simulations $[8,9,20]$ have examined the stabilizing effect of gravity $(\mathrm{Bo}>0)$ on $\mathrm{CF}$ and $\mathrm{VF}$, whereas its destabilizing effect $(\mathrm{Bo}<0)$ on VF is little documented.

Reference [16] investigated VF in 2D systems without gravity for values of $M \approx 10^{-4}$ and $0.36 \times 10^{-3}<\mathrm{Ca}<7.6$ $\times 10^{-3}$ (based on our definition of $\mathrm{Ca}$ in Sec. II). They observed qualitatively similar, treelike patterns and found that the growth of the structure is limited to an active zone in the vicinity of the most advanced finger. The analysis of the present experiments, as exemplarily shown in Fig. 5, reveals the additional influence of gravity in a destabilizing configuration: the growth is likewise confined to the uppermost fingers, but in addition a withdrawal of the NWF takes place in the lower area. This can be readily explained by the elevated hydrostatic pressure of the WF, which leads to a reduced capillary pressure $p_{c}=p_{\mathrm{NWF}}-p_{\mathrm{WF}}$ and therefore to the imbibition of the WF at the bottom.

\section{FLOW THROUGH THE POROUS MEDIUM}

After the characterization of the invasion processes in the previous section, the following discussions deal with the flow of the NWF through the porous medium-i.e., its dynamics after breakthrough at the upper boundary. Most studies on the dynamics of NWF aim at the characterization of
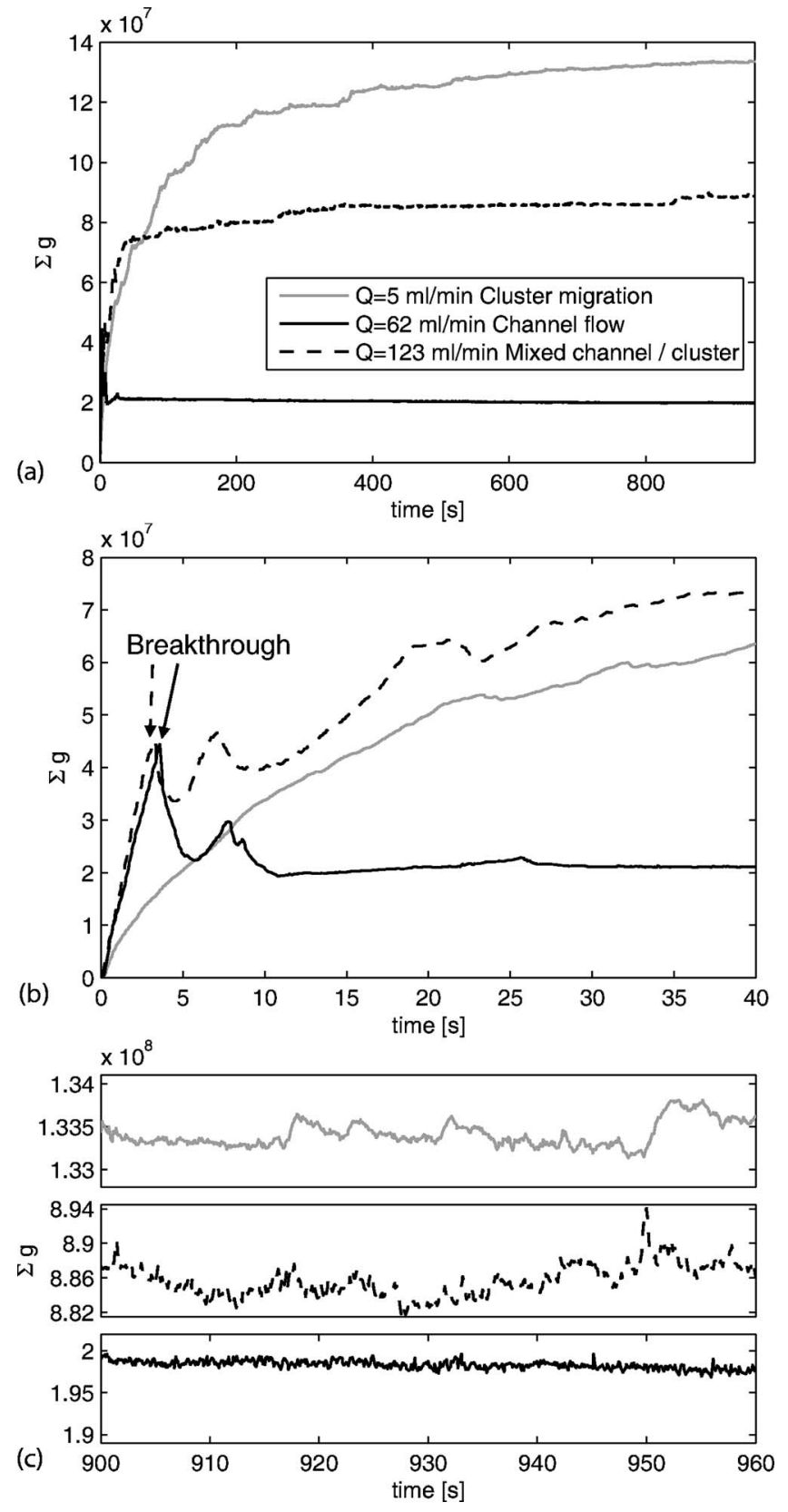

FIG. 4. Temporal evolution of total gas content $G(t)$ [Eq. (3)] for three experiments with $Q=5,62$, and $123 \mathrm{ml} / \mathrm{min}$, representing the regimes of cluster migration, single-channel flow, and mixed cluster and channel flow as shown in Fig. 6. (a) Full temporal range $t=0-1000 \mathrm{~s}$. (b) Initial behavior $t=0-40 \mathrm{~s}$. (c) Asymptotic behavior $t=900-1000 \mathrm{~s}$.

invasion processes. A few publications deal with the subsequent flow through a porous medium, like the advection through a percolation structure resulting, e.g., from $\mathrm{CF}$ [21-24]. In this case, only a small subset of the NWF, the so-called percolation backbone, contributes to the flow across the sample. For the present experiments, fundamentally differing behaviors have been observed mainly due to the additional influence of gravity.

The experiments described in the previous section have been continued with the same constant flow rates until an 


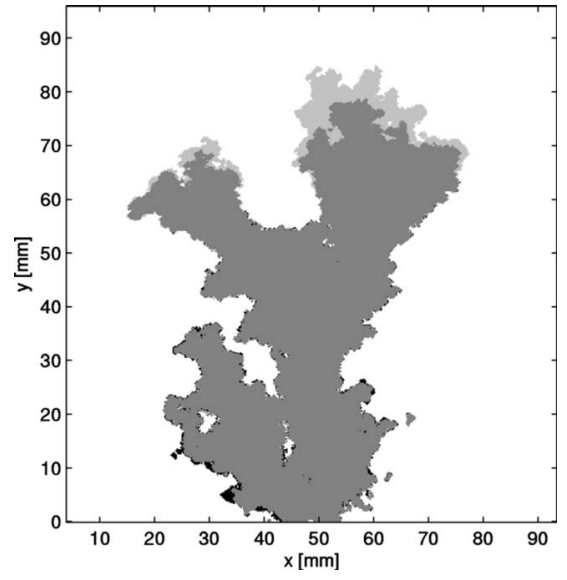

FIG. 5. Invasion of a fingerlike structure of gas (dark gray) for $Q=62 \mathrm{ml} / \mathrm{min}$. The picture has been compiled from two consecutive images separated by $\Delta t=0.3 \mathrm{~s}$. While the growth (light gray) is limited to the vicinity of the most advanced finger, the gas retracts (black) at the bottom due to the effect of hydrostatic pressure.

asymptotic behavior has been reached. The asymptotic distributions of gas for three different flow rates shown in Fig. 6 reveal the presence of distinct flow regimes for the respective flow rates.

In the following it will turn out that the capillary number (2) is no longer an appropriate quantity for the characterization of the observed patterns. Instead, the present phenomena are adequately represented by the combination of Bo with a new dimensionless number $K$, which represents the ratio of viscous and hydrostatic pressure gradients. On this basis, the phenomena can essentially be classified into two regimes: for $K>1$ the flow is predominantly localized in channels, and for $K<1$ the flow is characterized by the migration of isolated clusters. The mechanisms and features of these regimes will be discussed in the following subsections.

\section{A. Channel flow}

When the invasion of the connected fingerlike structures (see Sec. III B) reaches the upper boundary of the porous medium, the flow inside the connected NWF changes fundamentally. Whereas the velocities are perpendicular to the NWF-WF interface during the invasion, the perpendicular velocity components at the interface become zero for the flow through a stationary NWF distribution. In contrast to studies of the flow through percolation clusters, where it is implicitly assumed that the NWF distribution immediately becomes stationary at breakthrough [21-24], the connected NWF in the present configuration retracts after breakthrough due to the effect of hydrostatic pressure in the WF. The process of retraction is exemplified by the sequence of images shown in Figs. 2(b), 2(d), and 6(b) for an experiment with $Q=62 \mathrm{ml} / \mathrm{min}$. The asymptotic gas distribution in Fig. 6(b) shows that the fingerlike structure in Fig. 2(b) has essentially transformed into a single flow channel plus some residual trapped bubbles. This transition can also be identified from the temporal evolution of total gas content $G(t)$ plotted in Fig. 4. After the linear increase during invasion until breakthrough at $t=3.6 \mathrm{~s}$, the retraction is reflected by a decreasing gas content. This is followed by some temporary fluctuations, and finally a constant gas content is reached [Figs. 4(b) and $4(\mathrm{c})$ ] which indicates the formation of a stable channel.

The stability of this final configuration can be explained by the balance between hydrostatic pressures in the fluids and the viscous pressure in the gas channel as illustrated in Fig. 7 and discussed in the following.

The pressure of gas (NWF) in a vertical channel across the porous medium grows with depth as a result of both viscous and gravity forces,

$$
p_{\mathrm{NWF}}(y)=\left(\nabla p_{v}+\rho_{\mathrm{air}} g\right)(H-y)+\frac{2 \sigma}{d} .
$$

As a boundary condition the pressure at the upper boundary $p_{\mathrm{NWF}}(y=H)$ is equated to the capillary pressure $p_{\mathrm{c}}$ given by
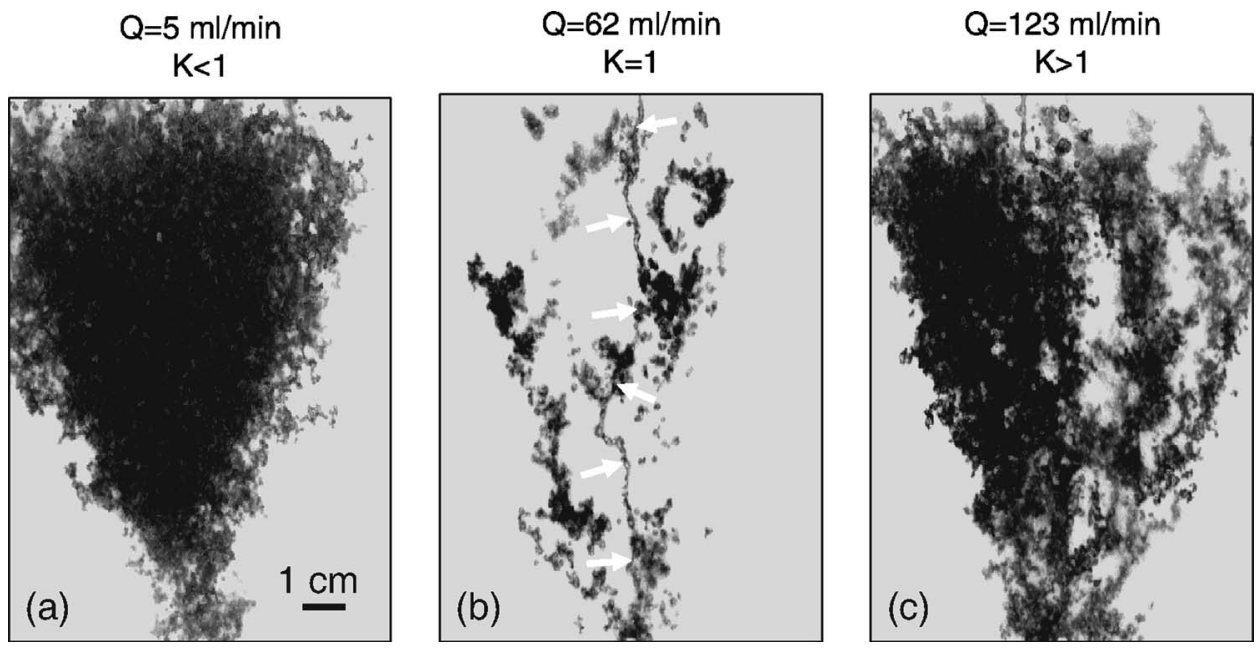

FIG. 6. Asymptotic distributions of gas after $t=500 \mathrm{~s}$ for three experiments with different flow rates. (a) For $Q=5 \mathrm{ml} / \mathrm{min}(K<1)$, the repeated fragmentation and trapping of isolated clusters gradually form a cone-shaped area of trapped bubbles. (b) For $Q=62 \mathrm{ml} / \mathrm{min}(K$ $=1$ ), the balance of hydrostatic and viscous pressure results in the flow through a stable vertical channel (indicated by white arrows). Additionally some remnants of the fingerlike invasion structure [Fig. 2(b)] are visible. (c) The dynamics for $Q=123 \mathrm{ml} / \mathrm{min}(K>1)$ is characterized by a combination of a stable channel and migrating clusters leaking out from the lower part of the channel. 


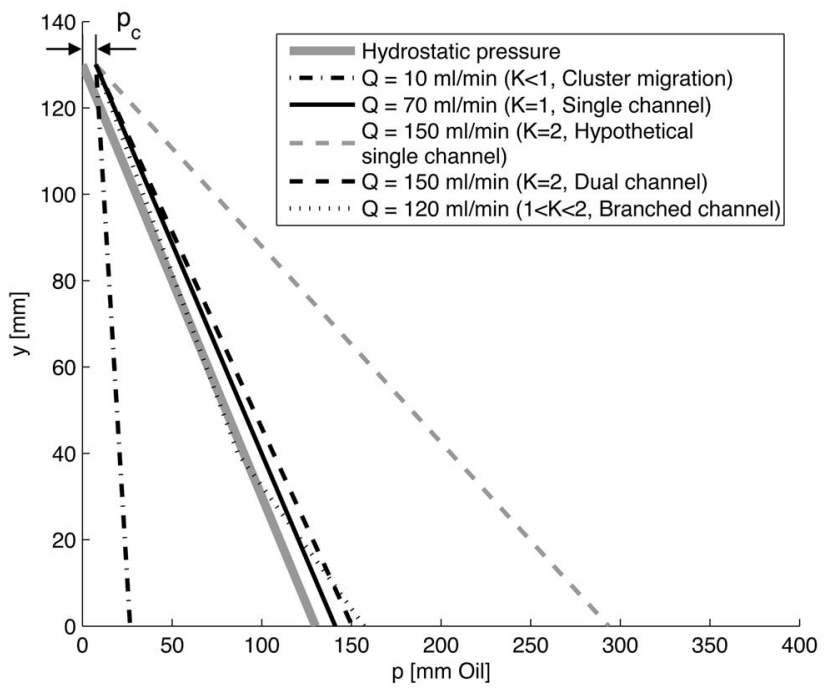

FIG. 7. Exemplary vertical distributions of hydrostatic and viscous pressures for the flow of gas through a channel in the porous medium. The viscous pressures are calculated for a channel diameter of $d=0.54 \mathrm{~mm}$ (with $p=p_{c}=2 \sigma / d$ at the upper boundary). For $Q=10 \mathrm{ml} / \mathrm{min}(K<1)$ and $Q=150 \mathrm{ml} / \mathrm{min}(K=2)$, the difference between hydrostatic and viscous pressure is too large and therefore a single channel is not stable. For $K<1$, this leads to a breakup into separated clusters, while for $K=2$, two separate channels form. Similar to the single $(Q=70 \mathrm{ml} / \mathrm{min}, K<1)$ and branched $(Q$ $=120 \mathrm{ml} / \mathrm{min}, 1<K<2$, branch at $y=40 \mathrm{~mm}$ ) channels, viscous and hydrostatic pressures are then compatible and therefore the channels are stable. Experimental manifestations of these phenomena are shown in Figs. 6 and 9.

Laplace's equation $p_{\mathrm{c}}=2 \sigma / d$. For the present range of Reynolds number $\operatorname{Re}=4 Q \rho_{\text {air }} / \pi d \mu_{\text {air }}, 8<\operatorname{Re}<540$ (for $d$ $=0.5 \mathrm{~mm}$ ), the viscous pressure gradient $\nabla p_{v}$ can be calculated from the law of Hagen and Poiseuille,

$$
\nabla p_{v}=\frac{128 Q \mu_{\text {air }}}{\pi d^{4}} .
$$

The pressure of the stagnant liquid (WF) in the porous medium grows with depth according to the hydrostatic pressure gradient,

$$
p_{\mathrm{WF}}(y)=\rho_{\mathrm{oil}} g(H-y) .
$$

Here the pressure is assumed to be zero at the upper boundary, $p_{\mathrm{WF}}(y=H)=0$.

The condition for the stability of the channel is now that the pressure difference between gas and liquid, $p_{\mathrm{NWF}}-p_{\mathrm{WF}}$ $=p_{\mathrm{c}}=2 \sigma / d$, is constant over depth. This is the case if the hydrostatic pressure gradient $\nabla p_{g}=\Delta \rho g\left(\Delta \rho=\rho_{\text {oil }}-\rho_{\text {air }}\right)$ is equal to the viscous pressure gradient $\nabla p_{v}$,

$$
\nabla p_{v} \stackrel{!}{=} \nabla p_{g}
$$

This is the basis for the definition of the dimensionless number $K$,

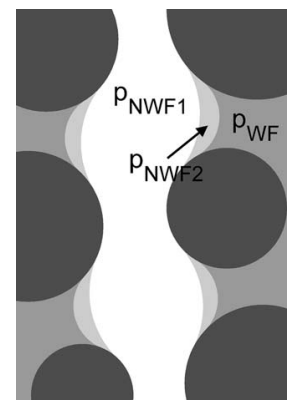

FIG. 8. Through the adaptability of the interface, the vertical channel of gas remains stable within a certain range of flow rates. The example shows an increase of the flow rate so that the capillary pressure $p_{\mathrm{c}}=p_{\mathrm{NWF}}-p_{\mathrm{WF}}$ increases by a factor of $2\left[p_{\mathrm{NWF} 2}-p_{\mathrm{WF}}\right.$ $\left.=2\left(p_{\mathrm{NWF} 1}-p_{\mathrm{WF}}\right)\right]$. This leads to a double curvature for $p_{\mathrm{NWF} 2}$ (light gray) compared to $p_{\mathrm{NWF} 1}$ (white).

$$
K=\frac{\nabla p_{v}}{\nabla p_{g}}=\frac{128 Q \mu_{\mathrm{air}}}{\pi d^{4} \Delta \rho g} .
$$

The requirement for the stability of the channel is then $K \stackrel{!}{=}$.

For the present experiments, the hydrostatic pressure gradient is $\nabla p_{g}=\Delta \rho g=9810 \mathrm{~kg} / \mathrm{ms}^{2}$. In these experiments the formation of stable channels occurs for flow rates $Q$ $>30 \mathrm{ml} / \mathrm{min}$. With that the requirement (7) leads to a value for the channel diameter of $d>0.44 \mathrm{~mm}$, which is reasonable for the given grain size distribution. A sketch of the corresponding pressure distributions is shown in Fig. 7. In practice the requirement (7) is not particularly strict, since the curvature of the NWF-WF interface and accordingly the capillary pressure can adapt over a certain range as shown in Fig. 8. If the pressure in the gas channel increases, the channel extents laterally, and the viscous pressure gradient decreases due to the larger channel cross section. Since $\nabla p_{v}$ scales with $d^{-4}$ according to Eq. (5), even a small increase of the channel diameter-say, by a factor of 1.3-leads to a considerable drop of the viscous pressure gradient by a factor of 3. This can explain that the formation of single channels $(K=1)$ has been observed over a wide range of flow rates $30 \mathrm{ml} / \mathrm{min}<Q<100 \mathrm{ml} / \mathrm{min}$ (corresponding to $0.44 \mathrm{~mm}$ $<d<0.59 \mathrm{~mm}$ ).

When the flow rate falls below $Q=30 \mathrm{ml} / \mathrm{min}(K<1)$, the excess of hydrostatic pressure in the WF leads to an imbibition and consequently to a breakup of the NWF channel. This implies the transition to a different regime characterized by the migration of isolated clusters as discussed in Secs. III $\mathrm{A}$ and IV B.

On the other hand, when the flow rate increases beyond a certain value, the excess pressure leads to a lateral escape of gas from the channel. As discussed above, flow through a single channel $(K=1)$ is stable up to $Q \approx 100 \mathrm{ml} / \mathrm{min}$. For an even higher $Q$, the flow divides into a corresponding number of channels. An approximately double flow rate $(Q$ $=186 \mathrm{ml} / \mathrm{min}$ ) leads therefore to the dual channel structure $(K=2)$ shown in Fig. 9(c). Figure 9(b) shows a branched channel $(1<K<2)$ which formed for the intermediate flow 

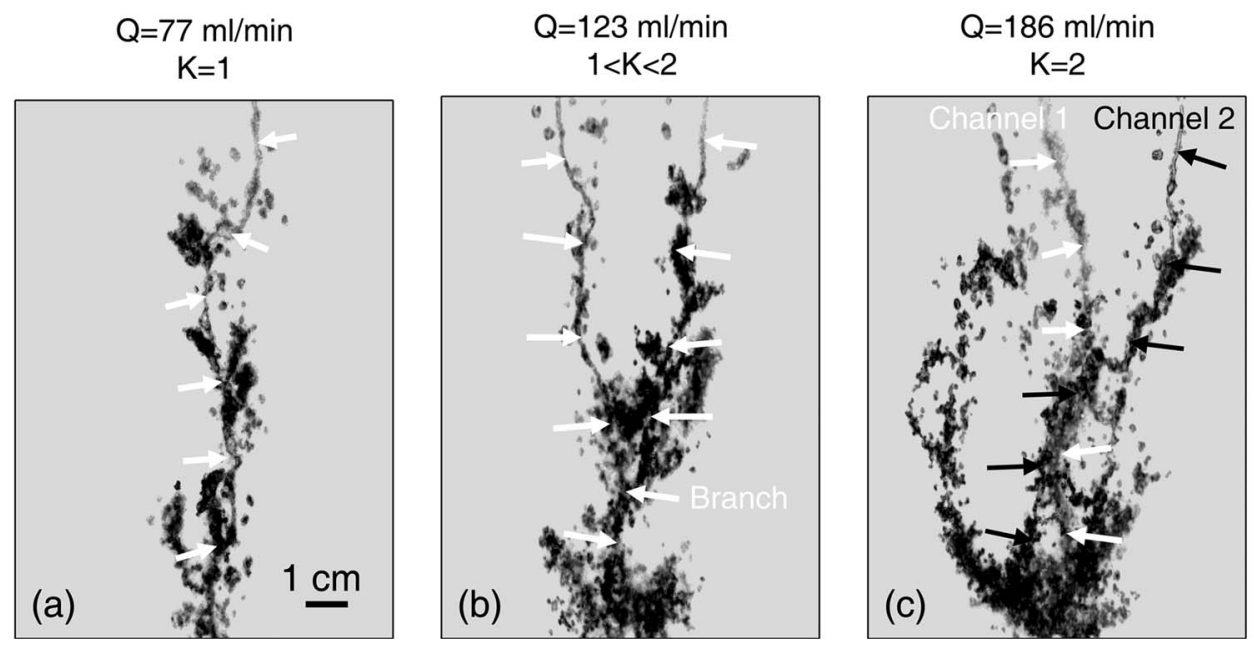

FIG. 9. Channel geometries for different gas flow rates. (a) Single channel for $Q=77 \mathrm{ml} / \mathrm{min}(K=1)$. (b) Branched channel for $Q$ $=123 \mathrm{ml} / \mathrm{min}(1<K<2)$. (c) Dual channel for $Q=186 \mathrm{ml} / \mathrm{min}(K=2)$. The flow paths of the stable channels are indicated by arrows.

rate $Q=123 \mathrm{ml} / \mathrm{min}$. These branched and divided channels are then again compatible with the required pressure balance as illustrated in Fig. 7. In some cases a mixed behavior-i.e., a combination of channel flow and cluster migration occurs as shown in Fig. 6(c). Here a constant leakage from the channel leads to a gradual formation of the cone-shaped area of trapped gas. Therefore, the temporal evolution of $G(t)$ plotted in Fig. 4(b) shows the characteristic peak at breakthrough $(t=3.3 \mathrm{~s})$, but then $G(t)$ gradually increases after breakthrough rather than becoming constant.

The essential mechanism for the stability of the observed channels is the local adaptability of the capillary forces as illustrated in Fig. 8. This leads to a local stabilization against minor fluctuations of channel diameter or flow rate. In a pure liquid without a porous matrix, where this mechanism is absent, such channels are unstable because small disturbances result in a breakup into bubbles. We have further made similar experiments with a more coarse-grained medium ( $d$ $\approx 2.5 \mathrm{~mm}, \mathrm{Bo} \approx-3)$. In this case it is impossible to reproduce any stable channel, and the gas always migrates in the form of isolated clusters. This makes sense because for $|\mathrm{Bo}|>1$ gravity dominates over capillary forces even on the pore scale.

\section{B. Cluster migration}

As discussed above, for $K<1(Q<30 \mathrm{ml} / \mathrm{min})$, the flow through a channel cannot be stable, and consequently the gas migrates upward through the porous medium in the form of isolated clusters. The comparison of the gas distributions in Figs. 2(a) and 2(c) indicates that this regime does not feature a pronounced breakthrough associated with an abrupt retraction of gas. Therefore, the temporal evolution of $G(t)$ shown in Figs. 4(a) and 4(b) does not involve a distinct peak, but a slow gradual increase towards an asymptotic maximum. This increase is caused by the repeated processes of fragmentation, trapping, blocking, and circumvention which have already been discussed in Sec. III A. More and more trapped gas accumulates with time in a cone-shaped area of the po- rous medium. Figure 6(a) exemplarily shows the distribution of gas at $t=5000 \mathrm{~s}$ for the experiment with $Q=5 \mathrm{ml} / \mathrm{min}$.

The temporal increase of $G(t)$ towards the asymptotic maximum plotted in Fig. 4(a) is not continuous but rather characterized by a steplike progression. This indicates that the cone-shaped area is not formed by the sequential drainage of individual pores, but by invasions of larger volumes as a whole, like, e.g., through the trapping of a migrating cluster. The phenomenon that invasion is not continuous but stepwise is a typical feature of drainage in porous media. Qualitatively similar behaviors have been reported previously in the literature for the process of slow-drainage 2D porous media [25-28], where the steplike invasions are commonly referred to as "Haines jumps."

Compared to the channel flow regime, the cluster migration is unstable in the sense that the paths of the upward moving gas permanently change. The asymptotic behavior, which is reached after a certain time, is equivalent to an equilibrium between trapping and remobilization of migrating clusters. The resulting fluctuations of $G(t)$ in this asymptotic regime [shown in Fig. 4(c)] are significantly stronger than those for the channel flow regime.

\section{SUMMARY AND CONCLUSIONS}

The flow of gas in a 3D unconsolidated porous medium under the combined effects of gravity, viscous, and capillary forces has been visualized using a light-transmission technique. The experiments have been characterized by a dimensionless number $K$, which represents the ratio of viscous and hydrostatic pressure gradients.

For low flow rates corresponding to $K<1$, the dynamics of gas is characterized by the migration of isolated clusters. The processes of fragmentation, trapping, blocking, and circumvention lead to a gradual formation of a cone-shaped area of trapped bubbles.

For high flow rates $(K>1)$, the gas invades the medium in the form of entirely connected structures which bear resemblance to patterns known from viscous fingering in $2 \mathrm{D}$ 
systems. The influence of hydrostatic pressure leads to simultaneous growth around the advancing tip and retraction at the bottom.

Directly after breakthrough at the upper boundary, the fingerlike invasion structure retracts under the influence of gravity and transforms into stable vertical channels. Steady flow of gas across the porous medium is then maintained through these channels. Depending on the value of $K$, different channel geometries evolve. It has been shown that the stability of these channels is the result of a balance between hydrostatic and viscous pressure gradients combined with the stabilizing effect of capillary forces.
The results demonstrate the essential influence of gravity for the dynamics of two-phase flow in 3D unconsolidated porous media. The findings are important for several applied issues since these conditions are present in most practical applications, like the injection of air into oil reservoirs for enhanced recovery or the migration of hydrocarbons in marine sediments.

\section{ACKNOWLEDGMENT}

M.S. acknowledges financial support from the Max Planck Society.
[1] J. Feder, Fractals (Plenum, New York, 1988).

[2] D. S. Stauffer and A. Aharony, Introduction to Percolation Theory, 2nd ed. (Taylor \& Francis, Philadelphia, 1994).

[3] T. Halpin-Healy and Y.-C. Zhang, Phys. Rep. 254, 215 (1995).

[4] S. Maslov, Phys. Rev. Lett. 74, 562 (1995).

[5] V. K. Horváth and H. E. Stanley, Phys. Rev. E 52, 5166 (1995).

[6] P. Meakin, Fractals, Scaling and Growth Far from Equilibrium (Cambridge University Press, Cambridge, England, 1998).

[7] M. Sahimi, Rev. Mod. Phys. 65, 1393 (1993).

[8] A. Birovljev, L. Furuberg, J. Feder, T. Jøssang, K. J. Måløy, and A. Aharony, Phys. Rev. Lett. 67, 584 (1991).

[9] Y. Méheust, G. Løvoll, K. J. Måløy, and J. Schmittbuhl, Phys. Rev. E 66, 051603 (2002).

[10] V. Frette, J. Feder, T. Jøssang, and P. Meakin, Phys. Rev. Lett. 68, 3164 (1992).

[11] P. Meakin, J. Feder, V. Frette, and T. Jøssang, Phys. Rev. A 46, 3357 (1992).

[12] D. Wilkinson, Phys. Rev. A 34, 1380 (1986).

[13] A. Birovljev, G. Wagner, P. Meakin, J. Feder, and T. Jøssang, Phys. Rev. E 51, 5911 (1995).

[14] G. Wagner, A. Birovljev, P. Meakin, J. Feder, and T. Jøssang, Phys. Rev. E 55, 7015 (1997).

[15] M. Stöhr, K. Roth, and B. Jähne, Exp. Fluids 35, 159 (2003).
[16] G. Løvoll, Y. Méheust, R. Toussaint, J. Schmittbuhl, and K. J. Måløy, Phys. Rev. E 70, 026301 (2004).

[17] H. Auradou, K. J. Måløy, J. Schmittbuhl, A. Hansen, and D. Bideau, Phys. Rev. E 60, 7224 (1999).

[18] A. C. Payatakes, Annu. Rev. Fluid Mech. 14, 365 (1992).

[19] P. Meakin, G. Wagner, J. Feder, and T. Jøssang, Physica A 200, 241 (1993).

[20] H. P. Huinink and M. A. J. Michels, Phys. Rev. E 66, 046301 (2002).

[21] J. S. Andrade, M. P. Almeida, J. Mendes Filho, S. Havlin, B. Suki, and H. E. Stanley, Phys. Rev. Lett. 79, 3901 (1997).

[22] H. A. Makse, J. S. Andrade, and H. E. Stanley, Phys. Rev. E 61, 583 (2000).

[23] A. Klemm, R. Kimmich, and M. Weber, Phys. Rev. E 63, 041514 (2001).

[24] J.-R. de Dreuzy, P. Davy, and B. Berkowitz, Phys. Rev. E 64, 056305 (2001).

[25] S. Roux and E. Guyon, J. Phys. A 22, 3693 (1989).

[26] K. J. Måløy, L. Furuberg, J. Feder, and T. Jøssang, Phys. Rev. Lett. 68, 2161 (1992).

[27] L. Furuberg, K. J. Måløy, and J. Feder, Phys. Rev. E 53, 966 (1996).

[28] E. Aker, K. J. Måløy, A. Hansen, and S. Basak, Europhys. Lett. 51, 55 (2000). 